\title{
A Evidência da Mamografia de Rastreio na Diminuição da Mortalidade por Cancro da Mama: Uma Revisão Sistemática
}

\section{The Evidence for Screening Mammography in Decreasing Breast Cancer Mortality: A Systematic Review}

Sara Laureano Alves ${ }^{1}$, Bernardo Pereira ${ }^{1}$, Isabel Peixoto ${ }^{1}$

Autor Correspondente:

Sara Laureano Alves [sara.laureanoalves@gmail.com] Rua de Ruães, n 19, 4710-565 Braga, Portugal

\section{RESUMO}

INTRODUÇÃO: O rastreio do cancro da mama é realizado através da mamografia nas mulheres. O objetivo desta revisão foi avaliar se a mamografia de rastreio diminui a mortalidade por cancro da mama.

MÉTODOS: Foi realizada uma pesquisa de artigos, publicados nos últimos 10 anos, através dos termos MeSH "mammography", "mortality", "mass screaning", "breast neoplasms". Para avaliação dos níveis de evidência e atribuição de forças de recomendação foi utilizada a escala Strenght of Recommendation Taxonomy.

RESULTADOS: Foram incluídos 15 artigos: três revisões sistemáticas, duas meta-análises, três estudos de coorte retrospetivos, quatro artigos de opinião, um estudo caso-controlo, uma revisão clássica e um ensaio clínico.

DISCUSSÃO: Os estudos analisados nesta revisão foram, na sua maioria, metodologicamente robustos e de boa qualidade, apontando para uma redução da mortalidade por cancro da mama mas não da mortalidade global. Assim, colocam em causa a continuidade dos programas de rastreio organizados de cancro da mama, constituindo esta uma força de recomendação A.

CONCLUSÃO: Parece consensual na literatura a evidência de sobrediagnóstico provocado pelos programas de rastreio cujas implicações não podem ser menosprezadas.

PALAVRAS-CHAVE: Mamografia; Mortalidade; Neoplasias da Mama; Programas de Rastreio 


\section{ABSTRACT}

INTRODUCTION: Breast cancer screening is performed through mammography in women. The purpose of this review is to determine if screening mammography decreases breast cancer mortality.

METHODS: A review of articles published in the last 10 years was done using the terms MeSH mammography, mortality, mass screening, breast neoplasms. The Strenght of Recommendation Taxonomy Scale was used to assess the levels of evidence and assignment recommendation forces.

RESULTS: Fifteen articles were included: three systematic reviews, two meta-analyzes, three retrospective cohort studies, four opinion articles, a case-control study, a classic review and a clinical trial.

DISCUSSION: The studies analyzed were mostly methodologically robust and with good quality, pointing to a reduction in breast cancer mortality but not from global mortality. Therefore, the continuity of organized breast cancer screening programs is jeopardized, constituting a recommendation force $A$.

CONCLUSION: The evidence of overdiagnosis caused by screening programs seems to be consensual in the literature, the implications of which can't be underestimated.

KEYWORDS: Breast Neoplasms; Mammography; Mass Screening; Mortality

\section{INTRODUÇÃO}

O rastreio do cancro da mama está implementado desde 2009 na região norte de Portugal, sendo a mamografia o teste de rastreio utilizado. Atualmente, o programa organizado é realizado em instalações próprias e conta com a colaboração da Liga Portuguesa Contra o Cancro (LPCC) e do Instituto Português de Oncologia (IPO). Trata-se de um rastreio de base populacional e tem como alvo mulheres com idades compreendidas entre os 45 e os 69 anos de idade. Caso não haja alterações que exijam uma vigilância mais apertada, a periodicidade do rastreio é bienal.

Um rastreio pressupõe que seja eficaz na redução da mortalidade e que seja custo-efetivo, em contraste com os possíveis falsos-positivos que dele possam advir. A mamografia é um exame que estuda o tecido mamário através de radiação ionizante, podendo detetar nódulos mamários indetetáveis à palpação. No entanto, pelo facto das alterações detetadas na mamografia não serem específicas de malignidade, o benefício da mamografia de rastreio começa a ser discutível devido à sua possível iatrogenia.

A mamografia de rastreio pode levar a um maior número de biópsias, ao sobrediagnóstico e ao sobretratamento (maior taxa de mastectomias e de radioterapia) com consequências físicas, psicológicas e financeiras desnecessárias. O tratamento por radioterapia pode aumentar a prevalência de enfarte agudo do miocárdio, por provocar lesões nas artérias coronárias, e aumentar a incidência de cancro do pulmão. Uma meta-análise sobre a radioterapia, como tratamento do cancro de mama, mostrou que há 27\% maior mortalidade por doença cardíaca e 78\% mais mortalidade por cancro de pulmão. Esta mortalidade torna-se importante quando mulheres saudáveis são sobrediagnosticadas. ${ }^{1}$

O impacto na diminuição da mortalidade por cancro da mama pela aplicação deste método de rastreio não é consensual em alguns países da Europa. Desta forma, é importante avaliar o custo-benefício da mamografia como método de rastreio populacional. A título de exemplo, um estudo realizado em Copenhaga (Dinamarca) concluiu que a aplicação da mamografia de rastreio requer uma ponderação dos seus riscos e benefícios. ${ }^{2}$ O Swiss Medical Board concluiu que a mamografia de rastreio não reduz a taxa de mortalidade por cancro da mama e recomendou a não introdução de novos programas de rastreio organizados e a suspensão gradual dos já implementados. O rastreio organizado deveria ser substituído por uma escolha individual baseada numa informação precisa e percetível dos seus riscos e benefícios. ${ }^{3}$ Nos EUA foi realizado um estudo que concluiu que para prevenir uma morte por cancro de mama em mulheres acima dos 50 anos de idade seria necessário rastrear 1000; que 0,3 a 3,2 mulheres vão evitar morte por cancro de mama; 490 a 670 mulheres vão ter pelo menos 1 falso alarme e que 3 a 14 vão ser sobrediagnosticadas e tratadas desnecessariamente. ${ }^{4}$

O objetivo desta revisão é responder à questão sobre o benefício do rastreio do cancro da mama por mamografia na redução da sua mortalidade.

\section{MÉTODOS}

Realizámos, durante o mês de junho de 2017, uma pesquisa de normas de orientação clínica (NOC), revisões sistemáticas (RS), meta-análises e ensaios clínicos alea- 
torizados e controlados, publicados entre junho de 2007 e junho de 2017. As bases de dados utilizadas foram a PubMed, Cochrane, Dare, Bandolier, NHS e a National Guideline Clearinghouse. Os termos MeSH (medical subject headings) utilizados foram: "Mammography", "Mortality", "Mass Screening" e "Breast Neoplasms".

Os critérios utilizados para a inclusão dos artigos nesta revisão foram os seguintes:

- População: mulheres entre os 45 e 69 anos de idade, assintomáticas, sem história pessoal de cancro da mama;

- Intervenção: realização de mamografia de rastreio;

- Comparação: mamografia versus sem mamografia;

- Resultados: diminuição da mortalidade por cancro da mama;

- Foram apenas incluídos artigos em língua portuguesa e inglesa.

Para avaliação dos níveis de evidência e atribuição de forças de recomendação foi utilizada a escala Strenght of Recommendation Taxonomy (SORT): nível A (ensaios clínicos aleatorizados controlados/meta-análises); nível B (outra evidência) e nível C (consensos/opinião de peritos). 5

\section{RESULTADOS}

Da pesquisa que efetuámos foram encontrados 123 artigos. Destes, foram excluídos os artigos repetidos, aqueles em que se verificou discordância com o objetivo da revisão e aqueles que não cumpriam os critérios de inclusão previamente descritos. Foram também excluídos estudos históricos, isto é, estudos em que eram comparados resultados de populações de diferentes épocas. Nesta sequência obtivemos 15 artigos: três RS, duas meta-análises (MA), três estudos de coorte retrospetivos (CR), quatro artigos de opinião (AO), um estudo caso-controlo (CC), uma revisão clássica (RC) e um ensaio clínico (EC).

Após a leitura dos artigos incluídos agrupámos os resultados em dois tópicos para melhor compreensão. As conclusões de todos os artigos analisados encontram-se detalhadas nas Tabelas 1 a 6 .

1) Impacto da mamografia de rastreio na diminuição da mortalidade por cancro da mama contra a não realização da mamografia de rastreio.

Com exceção do ensaio clínico Canada 1980 incluído na RS Gøtzsche PC et al, ${ }^{6}$ no qual não houve diferenças estatisticamente significativas na mortalidade entre o grupo submetido a mamografia de rastreio e o grupo controlo, as revisões sistemáticas constataram menor mortalidade por cancro da mama no grupo de mulheres rastreadas. Este resultado também foi constatado em todos os estudos de coorte incluídos. No entanto, não houve impacto significativo na mortalidade global.

No estudo caso-controlo Miller AB et al ${ }^{7}$ não se verificou diferenças na mortalidade provocada pela doença entre os 2 grupos.

Ann Johnson, 8 na sua revisão clássica, concluiu que a mamografia favorece o diagnóstico de tumores de crescimento lento, pelo que o potencial ganho em mortalidade é reduzido.

O artigo de opinião de Susan Mayor incluiu uma análise de dados de uma amostra de mulheres suecas, a qual demonstrou pouco ou nenhum efeito da mamografia de rastreio na mortalidade por cancro da mama. Os restantes artigos de opinião incluídos ${ }^{1,10}$ contrariam esta ideia, tendo demonstrado redução entre 15\% a 65\% na mortalidade nas mulheres rastreadas.

Os estudos Hakama e Peer, incluídos na meta-análise de Irvin VL et al,11 não demonstraram impacto na mamografia de rastreio, enquanto que nos restantes quatro estudos incluídos nesta meta-análise verificaram redução da mortalidade.

Por último, o ensaio clínico de Tabár L ${ }^{12}$ corrobora o impacto positivo da mamografia, demonstrando uma redução de 30\% na mortalidade no grupo rastreado.

2) Sobrediagnóstico e sobretratamento das mulheres submetidas a mamografia de rastreio.

Em todas as RS incluídas, o grupo de mulheres submetidas a mamografia de rastreio esteve associado a uma maior taxa de sobrediagnóstico (com um maior número de biópsias mamárias realizadas) e, consequentemente, sobretratamento (mastectomias e radioterapia).

No estudo caso-controlo de Miller $\mathrm{AB}^{7}$ foi detetada uma taxa de 22\% de sobrediagnóstico e sobretratamento, ou seja, houve um caso de sobrediagnóstico em cada 424 mulheres submetidas a mamografia de rastreio.

Na RC de Johnson $A^{8}$ é descrito que existe sobrediagnóstico e sobretratamento dos carcinomas in situ, o que se traduz apenas num pequeno aumento da esperança média de vida. As populações rastreadas e não rastreadas não devem ser comparadas porque os rastreios alteram a apresentação da doença.

Nos artigos de opinião de Jørgensen $\mathrm{KJ}$ et al ${ }^{1}$ e Kopans DB et $a^{10}$ é referido que o sobrediagnóstico e o sobretratamento são inevitáveis, contudo estas estimativas são difíceis de calcular. 
TABELA 1. Revisões sistemáticas e estudos de coorte retrospetivos incluídos na revisão.

\section{REVISÕES SISTEMÁTICAS}

Gøtzsche PC, Jørgensen KJ (2013)

$$
\begin{aligned}
& \text { Métodos } \\
& \text { População } \\
& \mathrm{N}=5 \text { estudos } \\
& \text { - Canadá } 1980 \\
& n=89931 \\
& \text { M entre } 40-59 \text { anos } \\
& \text { - Göteborg } 1982 \\
& n=52222 \\
& \text { M entre } 39-59 \text { anos } \\
& \text { - New York } 1963 \\
& n=62184 \\
& \text { M entre } 40-64 \text { anos } \\
& \text { - Stockholm } 1981 \\
& n=\text { inconsistente } \\
& \text { M entre 40-64 anos } \\
& \text { Two-County } 1977 \\
& n=220878 \\
& \text { Mentre 40-74 anos }
\end{aligned}
$$

\section{Intervenção}

Mamografia de rastreio

de cancro da mama

\section{Resultados}

Impacto da mamografia de rastreio na diminuição da mortalidade por cancro da mama contra a não realização da mamografia de rastreio

Canada 1980: sem diferenças estatisticamente significativas na mortalidade entre o grupo submetido a rastreio e o grupo controlo.

Göteborg 1982, New York 1963, Stockholm 1981, TwoCounty 1977: concluíram menor mortalidade por cancro da mama no grupo de mulheres submetidas a mamografia de rastreio, embora não se tenha traduzido num aumento da sobrevida global.

\section{Sobrediagnóstico e sobretratamento das mulheres submetidas a mamografia de rastreio}

Canada 1980: verificou-se sobretratamento (cirurgia) no grupo de mulheres rastreadas com mamografia após 7 anos de seguimento.

Stockholm 1981: verificaram existir mais 25\% de mastectomias no grupo rastreado.

Göteborg 1982, New York 1963, Stockholm 1981, TwoCounty 1977: concluíram que houve sobretratamento nas mulheres que realizaram mamografia de rastreio (estas mulheres foram submetidas a mais tratamentos de radioterapia).

Nos outros ensaios clínicos randomizados incluídos nesta revisão constatou-se mais 30\% de sobrediagnósticos, mais 30\% de cirurgias e mais 20\% de mastectomias. O risco cumulativo de um falso-positivo após 10 mamografias varia de $20 \%$ a $60 \%$.

\section{Conclusão}

Não houve evidência do efeito benéfico do rastreio por mamografia na taxa de mortalidade por cancro da mama.

Broeders M, Moss S, Nystrom L, et al (2012) ${ }^{13}$

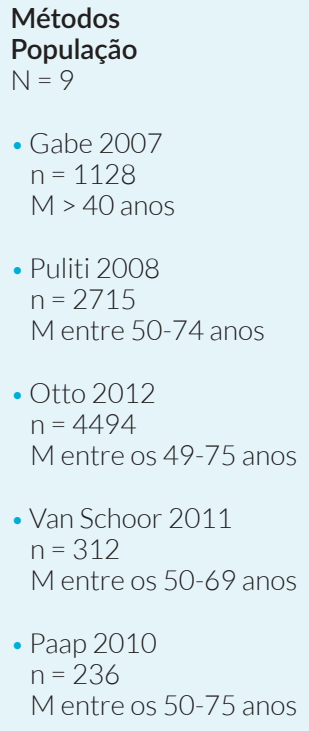

\section{Resultados}

Impacto da mamografia de rastreio na diminuição da mortalidade por cancro da mama contra a não realização da mamografia de rastreio

Gabe 2007: o risco relativo de morte por cancro da mama nas mulheres submetidas a mamografia foi menor comparativamente com as mulheres que não foram submetidas a exame de rastreio (RR 0,59 vs RR 1).

Puliti 2008: o odds-ratio comparando mulheres submetidas a mamografia de rastreio com as que não foram submetidas foi de 0,5 (95\% Cl: 0,42-0,59). Não houve variação significativa no odds-ratio entre os grupos com diferentes faixas etárias.

Otto 2012: relata que a mamografia de rastreio está associada à diminuição do risco de cancro da mama e que este efeito é cumulativo, ou seja, é maior nas mulheres que foram submetidas a 3 ou mais mamografias de rastreio.

Van Schoor 2011: demonstrou impacto significativo da mamografia na mortalidade por cancro da mama ao longo do tempo. As mulheres que realizaram o rastreio tiveram uma taxa de mortalidade 35\% inferior às não rastreadas.

Paap 2010: redução na mortalidade de 76\%, após ajuste para possível viés de seleção, por cancro da mama nas mulheres submetidas a mamografia de rastreio comparativamente com as não rastreadas. 
Métodos (cont.)
- Allgood 2008
$n=852$
M entre os 50-70 anos
- Sarkeala 2008
$n=2338775$
M entre os 50-69 anos
- Hakama 1997
$n=158755$
M entre os $45-69$ anos
- Paci, Eur J Cancer 2002
$n=113$
M entre os 50-69 anos

Intervenção

Mamografia de rastreio de cancro da mama

\section{Nelson HD, Tyne K, Naik A, et al (2009)14}

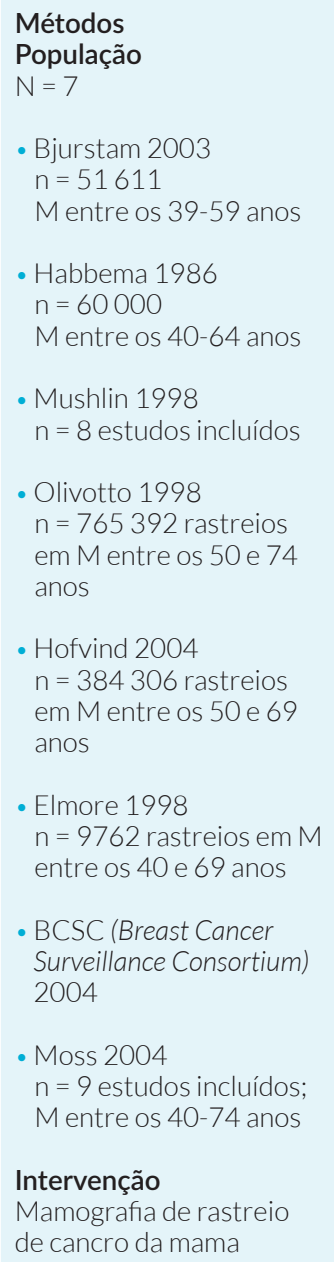

Resultados (cont.)

Allgood 2008: redução de 48\% de mortalidade por cancro da mama, nas mulheres rastreadas. Este artigo poderá ter um ligeiro viés a favor do rastreio por selecionar mulheres recentemente diagnosticadas com cancro da mama que provavelmente terão cancros mais agressivos e com rápida progressão.

Sarkeala 2008: nos municípios com rastreio organizado, as mulheres com idades entre os 50-69 anos tiveram uma diminuição de $28 \%$ na mortalidade com significância estatística (0,72, 0,51-0,97).

Hakama 1997: demonstrou um efeito protetor de $24 \%$ na redução da mortalidade, mas que não era significativo. Houve diminuição da mortalidade estatisticamente significativa $(0,56 ; 0,33-0,95)$ apenas nas mulheres com idade superior a 56 anos no início do rastreio; foi prevenida uma morte por cada 10000 rastreios.

Paci, Eur J Cancer 2002: os programas de rastreio organizado têm pouco impacto na mortalidade por cancro da mama nos primeiros 10 anos da sua implementação.

\section{Sobrediagnóstico e sobretratamento das mulheres submetidas a mamografia de rastreio}

Nos vários artigos analisados, não foram encontrados dados referentes a este ponto.

\section{Conclusão}

Houve uma diminuição da mortalidade por cancro da mama superior a 28\% nas mulheres europeias com idades compreendidas entre os 45 e os 69 anos. Parece existir um efeito cumulativo, isto é, quantos mais anos de rastreio maior é o seu efeito benéfico na mortalidade por cancro da mama.
Impacto da mamografia de rastreio na diminuição da mortalidade por cancro da mama contra a não realização da mamografia de rastreio

Bjurstam 2003 (Gothenburg trial): o RR da mortalidade por cancro de mama é 0,85 (95\% Crl 0,75-0,96) nas mulheres rastreadas e o NNI para o rastreio para prevenir uma morte por cancro de mama é 1.904 (95\% Crl, 929-3378).

Habbema 1986: o resultado indica um efeito benéfico do rastreio na mortalidade por cancro de mama. A diminuição da mortalidade do grupo rastreado, quando comparado ao grupo controlo, foi significativa independentemente do tempo de inclusão (5,7 e 10 anos após início) e seguimento (5,10 e 14 anos).

Abaixo dos 50 anos não foi observada diferença na mortalidade, ao contrário do que aconteceu acima dessa idade.

\section{Sobrediagnóstico e sobretratamento das mulheres submetidas a mamografia de rastreio}

Mushlin 1998: a probabilidade de falsos-positivos da mamografia de rastreio foi estimada em 0,9\%-6,5\%.

Olivotto 1998, Hofvind 2004, Elmore 1998: o risco cumulativo de falsos-positivos na mamografia foi de 21\%-49\% após 10 mamografias nas mulheres em geral e mais de 56\% nas mulheres entre os 40-49 anos.

BCSC 2004: para deteção de 1 caso de cancro de mama invasivo através da mamografia de rastreio em mulheres entre os 40-49 anos é necessário que 556 mulheres realizem mamografia; que sejam realizados 46-48 métodos de imagem adicionais e 5-8 biópsias. Com o aumento da idade, estes resultados diminuem em número para a mamografia e para os métodos de imagem adicionais, verificando-se apenas uma ligeira diminuição do número de biópsias.

Moss 2004: a incidência cumulativa do cancro da mama invasivo e não-invasivo atribuída ao sobrediagnóstico em mulheres submetidas a mamografia de rastreio foi de 0,07-0,73 por 1000 mulheres/ano.

\section{Conclusão}

As mulheres mais jovens (entre os 40-49 anos) são as que têm maior probabilidade de falsos-positivos, isto é, são as que estão mais sujeitas a sobrediagnóstico e consequentemente sobretratamento. 


\section{ESTUDO DE COORTE RETROSPETIVO}

Kaplan HG, Malmgren JA, et al (2015) ${ }^{15}$

\section{Métodos}

População

Mulheres entre os 50

e os 69 anos que foram

diagnosticadas com

cancro de mama entre

1990 e 2007

$(n=52998)$

\section{Intervenção}

Mulheres com cancro de mama diagnosticado por mamografia versus por exame físico

\section{Resultados}

Resultados foram divididos em 3 grupos, conforme o ano de diagnóstico do cancro de mama (entre 19901994; 1995-1999 e 2000-2007). Houve diferenças estatisticamente significativas, entre os 3 grupos, relativamente à sobrevida após 5 anos de diagnóstico, com taxa mais alta no período de tempo 20002007 (97\%) vs $\mathbf{9 6 \%}$ entre 1995 -1999 e $\mathbf{9 1 \%}$ entre 1990-1994, justificadas possivelmente por mudanças na apresentação, diagnóstico e tratamento do cancro de mama.

A mamografia aumentou a deteção de cancros em 52\% de 1990-1994 e 61\% no período entre 20002007.

Impacto da mamografia de rastreio na diminuição da mortalidade por cancro da mama contra a não realização da mamografia de rastreio

De acordo com o método de deteção do cancro de mama, mulheres com diagnóstico por mamografia apresentaram uma diminuição significativa de morte provocada por doença após 5 anos (RR, 0,43; IC [95\%] 0,27-0,70)

\section{Sobrediagnóstico e sobretratamento das mulheres submetidas a mamografia de rastreio}

O artigo não tem dados referentes a este ponto.

\section{Conclusão}

Deteção de cancro de mama por mamografia está associada a uma diminuição de 57\% da mortalidade provocada pela doença.

Hofvind S, Ursin G, et al $(2013)^{16}$

\section{Métodos \\ População}

Mulher entre os 50-69

anos, sem diagnóstico prévio de cancro de mama, convidadas para mamografia de rastreio entre 1996 e 2009

$(n=699628)$

\section{Intervenção}

Mulheres submetidas a versus mulheres que não realizaram mamografia (não responderam à convocatória de rastreio) rastreio de mamografia

\section{Resultados}

Taxa de mortalidade atribuída ao cancro de mama foi de 20,7 por 100000 "anos sem doença" no grupo das mulheres submetidas a mamografia de rastreio e de 39,7 por 100000 no grupo das mulheres não rastreadas.

Constatou-se um aumento estatisticamente significativo na taxa de mortalidade de acordo com o aumento da idade e dos anos de seguimento.

Impacto da mamografia de rastreio na diminuição da mortalidade por cancro da mama contra a não realização da mamografia de rastreio

Após 15 anos de seguimento, verificou-se uma redução de 43\% na taxa de mortalidade por cancro de mama no grupo das mulheres submetidas a mamografia de rastreio, comparativamente com o grupo não rastreado. Após correção para possível viés, a redução na taxa de mortalidade verificada é cerca de $25 \%$ no grupo rastreado com mamografia.

\section{Sobrediagnóstico e sobretratamento das mulheres submetidas a mamografia de rastreio}

O artigo não tem dados referentes a este ponto.

\section{Conclusão}

Nas mulheres entre os 50 e os 69 anos que foram convidadas para rastreio bienal do cancro de mama houve uma redução substancial da mortalidade pela doença no grupo rastreado, comparativamente com as não rastreadas.

Kalager M, Zelen M, Langmark F, et al (2010) ${ }^{17}$

\section{Métodos}

População

- $n=40075$

M 50-69 anos

Seguimento de 8,9 anos

\section{Intervenção}

Mamografia de rastreio de cancro da mama

\section{Resultados}

Impacto da mamografia de rastreio na diminuição da mortalidade por cancro da mama contra a não realização da mamografia de rastreio

Redução de 10\% de mortalidade por cancro de mama no grupo de mulheres que realizaram mamografia.

\section{Sobrediagnóstico e sobretratamento das mulheres submetidas a mamografia de rastreio}

O artigo não tem dados referentes a este ponto.

\section{Conclusão}

Os resultados sustentam a evidência de que a mamografia de rastreio reduz a taxa de mortalidade por cancro de mama. No entanto, a magnitude desse benefício parece ser modesta no programa de triagem nacional testado.

(RR - risco relativo; $\mathrm{Crl}$ - intervalo de confiança; NNI - number need to invite (número necessário a convidar); $\mathrm{M}$ - mulheres; $\mathrm{n}$ - tamanho da amostra 
TABELA 2. Estudo de caso-controlo incluído na revisão.

\section{ESTUDOS CASO-CONTROLO}

Miller AB, Wall C, Baines CJ, et al (2014)

\section{Métodos}

\section{População}

Mulheres entre os 40-59 anos não grávidas, que não tenham realizado mamografia nos últimos 12 meses e sem antecedentes pessoais de cancro de mama

$(n=89835)$

Todas as participantes receberam treino da técnica de auto-palpação mamária por enfermeiros

\section{Intervenção}

Mulheres submetidas a mamografía + exame físico (anual) versus mulheres submetidas a exame físico mamário anual sem realização de mamografia

\section{Resultados}

A sobrevida aos 25 anos das mulheres com cancro de mama palpável diagnosticados foi semeIhante nos dois grupos (66,3\% vs 62,8\%).

Impacto da mamografia de rastreio na diminuição da mortalidade por cancro da mama contra a não realização da mamografia de rastreio

A mortalidade cumulativa global e por cancro da mama, aos 25 anos de seguimento, foram semelhantes entre o grupo de mulheres submetidas a mamografia e o grupo que não realizou mamografia $(p=0,28 ; p=0,87)$

\section{Sobrediagnóstico e sobretratamento das mulheres submetidas a mamografia de rastreio}

Dos cancros de mama detetados por mamografia, 22\% são sobrediagnosticados. Isto significa que houve um caso de sobrediagnóstico em cada 424 mulheres que receberam mamografia de rastreio.

\section{Conclusão}

A realização de mamografia de rastreio anual (programa com 5 mamografias anuais consecutivas) não reduz a mortalidade por cancro de mama, em mulheres com idades compreendidas entre 40 e 59 anos.

(n - número de artigos de opinião)

TABELA 3. Revisão clássica incluída na revisão.

\section{REVISÃO CLÁSSICA}

Johnson A (2012)

\section{Métodos}

População

Revisão clássica

\section{Resultados}

As neoplasias têm um crescimento exponencial do seu volume, não sendo este um crescimento linear e igual em todos os casos. Tumores com mais de $10 \mathrm{~mm}$ podem ser diagnosticados clinicamente.

A mamografia de rastreio altera a apresentação do cancro de mama, favorecendo o diagnóstico de tumores de crescimento lento. Assim, o potencial ganho em termos de mortalidade é pequeno e difícil de demonstrar.

Sobrediagnóstico e sobretratamento das mulheres submetidas a mamografia de rastreio

O aumento muito pequeno da esperança média de vida é conseguido através de um sobretratamento de carcinomas in situ e de tumores de crescimento lento.

TABELA 4. Artigos de opinião incluídos na revisão.

\section{ARTIGOS DE OPINIÃO}

Burke JP, Barry M, Kell MR, et al $(2011)^{18}$

\section{Métodos}

$\mathrm{n}=5$ artigos de

opinião

Sobre o artigo

de Kalager et al

\section{Resultados}

Impacto da mamografia de rastreio na diminuição da mortalidade por cancro da mama contra a não realização da mamografia de rastreio

Burke JP, Barry M, Kell MR: foi encontrada uma redução de 10\% de mortalidade no grupo de mulheres que realizou a mamografia de rastreio, mas apenas foram contabilizadas as causas de morte relacionadas ao cancro e não todas as causas de morte. É consensual que a avaliação de todas as causas de morte é mais válida na deteção do efeito do rastreio do cancro de mama.

Foi encontrada uma discrepância no grupo de mulheres com menos de 50 anos que realizaram a mamografia: Kalager et al, encontrou um aumento da mortalidade em 4\%, mas os dados da OMS demonstram uma redução de 35\% da mortalidade nesse grupo.

Os autores levantam a hipótese de que a redução na mortalidade encontrada por Kalager et al poderá ser devida ao facto da introdução de um programa de rastreio aumentar a conscientização acerca do cancro de mama, o que leva a um aumento do autoexame e consequentemente à mamografia realizada de forma oportunista.

Os autores Ravesteyn NT, Heijnsdijk EAM, Koning HJ levantam a hipótese de que a abordagem de Kalager et al, embora bem executada, subestime consideravelmente o efeito verdadeiro da mamografia de rastreio por usar um período de seguimento muito reduzido.

Duffy SW, Smith RA: os autores consideram que a pequena redução da mortalidade no grupo de mulheres rastreadas se deve a um período de seguimento demasiado curto.

Fletcher SW, Elmore JG: quando o tratamento é muito eficaz, independentemente da presença de sintomatologia, o rastreio deixa de ter um papel importante. No cancro de mama a eficácia demonstrada dos tratamentos tornam a recomendação do rastreio como algo incerto. Para além do artigo de Kalager et al, resultados de mortalidade a 10 anos não foram estatisticamente significativos num estudo randomizado mais recente, realizado no Reino Unido, com mortalidade inferior ao esperado no grupo não examinado (Moss SM et al); e um estudo caso-controlo baseado na população nos Estados Unidos em 2005 também não mostrou nenhum efeito significativo no rastreio de cancro da mama (Elmore JG et al).

Sobrediagnóstico e sobretratamento das mulheres submetidas a mamografia de rastreio O artigo não tem dados referentes a este ponto.

NE 
Mayor S (2012)

$\mathrm{n}=1$ artigo de

Resultados

Impacto da mamografia de rastreio na diminuição da mortalidade por cancro da mama contra a não realização

Uma análise de dados suecos demonstrou que a mamografia de rastreio tem pouco ou nenhum efeito na mortalidade por cancro da mama em mulheres dos 40 aos 69 anos.

Os resultados da investigação liderada por Philippe Autier demonstraram que a mortalidade por cancro da mama começou a diminuir antes da introdução da mamografia de rastreio e a taxa de diminuição manteve-se sempre similar no período pré e pós-rastreio.

Michael Vannier, argumentou que as limitações do tratamento têm uma maior influência na mortalidade por cancro da mama do que prováveis "falhas" do rastreio. Ele recomendou a implementação do rastreio por subgrupos de acordo com o risco e sugeriu que melhores ferramentas de diagnóstico ajudariam a equilibrar o custo-benefício do rastreio.

Para Nereo Segnan e colaboradores, já não é razoável o debate em torno dos prováveis benefícios do rastreio através da mamografia, como se este estivesse isolado do restante serviço de saúde. As prioridades devem centrar-se em aumentar a acuidade do rastreio, mas também a eficácia do tratamento.

Sobrediagnóstico e sobretratamento das mulheres submetidas a mamografia de rastreio

$\bigcirc$ artigo não tem dados referentes a este ponto.

Jørgensen KJ, Keen JD, Gøtzsche PC (2011)

$\mathrm{n}=1$ artigo de

opinião

\section{Resultados}

Impacto da mamografia de rastreio na diminuição da mortalidade por cancro da mama contra a não realização da mamografia de rastreio

Os defensores do rastreio mamográfico argumentam que este diminui a mortalidade por cancro da mama em cerca de 30\%. Nesta perspetiva otimista inclui-se o estudo "The Swedish Two-County Trial". No entanto, revisões sistemáticas levadas a cabo pelo US Preventive Services Task Force e pelo Nordic Cochrane Centre sugeriram uma redução de apenas 15\%-16\%.

Neste artigo é também realçado que houve muitos avanços no tratamento do cancro da mama desde a época em que a maioria dos ensaios clínicos foram realizados, e esses avanços reduziram o efeito do rastreio.

Para Daniel Kopans, se uma intervenção de rastreio fosse efetiva, deveria notar-se uma redução substancial das neoplasias avançadas. No entanto, o que se verificou com a implementação do rastreio foi um grande aumento do carcinoma ductal in situ e dos cancros de mama localmente invasivos e uma discreta redução dos cancros de mama metastizados. Isto foi relatado em várias revisões sistemáticas de vários países.

Em contraste com o rastreio, o aumento da consciência/preocupação com o cancro da mama foi um fator importante que levou à diminuição da mortalidade por cancro da mama. Na Dinamarca, o tamanho do tumor tinha em média 33 mm em 1978, mas apenas 24 mm dez anos mais tarde, tendo esta transformação acontecido antes da introdução do rastreio.

Existem muitos estudos observacionais mal desenhados que afirmam existir um grande efeito da mamografia de rastreio na mortalidade por cancro da mama. Exemplos desses estudos foram os realizados na Dinamarca, Reino Unido, Suécia, Noruega e Estados Unidos.

\section{Sobrediagnóstico e sobretratamento das mulheres submetidas a mamografia de rastreio}

O sobrediagnóstico é inevitável. Muitas mulheres morrem por outras causas antes do seu cancro da mama (detetado pelo rastreio) ser clinicamente evidente.

No Malmö Mammographic Screening Trial, a taxa de sobrediagnóstico foi de 10\%, mas depois de ajustados para os possíveis vieses foi de 25\%. Uma revisão da Cochrane reportou 30\% de sobrediagnóstico, que corresponde a 10 casos de sobrediagnóstico por cada vida prolongada. Noutras revisões sistemáticas foi encontrada uma taxa de $52 \%$ de sobrediagnóstico.

Vários ensaios clínicos demonstraram que a taxa de mastectomias era 20\% superior nas mulheres submetidas a rastreio e estudos observacionais demonstraram um aumento deste número. Este aumento refuta o mito que um diagnóstico precoce leva a uma diminuição das mastectomias realizadas.

\section{Conclusão}

O sobrediagnóstico e o sobretratamento aumentam a mortalidade das mulheres rastreadas e isto deve ser tido em conta. É importante relembrar que é sempre melhor prevenir o cancro do que detetar precocemente. $\mathrm{O}$ US Center for Medical Consumers defende que se quisermos reduzir a incidência do cancro da mama não há nada tão efetivo como evitar a mamografia de rastreio.

Kopans DB, Smith RA, Duffy SW $(2011)^{10}$

\section{$n=1$ artigo de} opinião

\section{Resultados}

Impacto da mamografia de rastreio na diminuição da mortalidade por cancro da mama contra a não realização da mamografia de rastreio

Investigadores estimaram que a mamografia foi responsável por 28\% a 65\% de declínio na taxa de mortalidade por cancro da mama.

\section{Sobrediagnóstico e sobretratamento das mulheres submetidas a mamografia de rastreio}

Jørgensen e Gøtzsche concluíram, no seu estudo, que há elevadas taxas de sobrediagnóstico nos programas de rastreio, no entanto, este estudo tem graves erros metodológicos.

Zahl et al realizou um estudo histórico e, por causa deste erro metodológico, concluiu que houve sobrediagnóstico com a mamografia de rastreio (a incidência do cancro da mama aumentou durante as décadas englobadas pelo es tudo).

Estimar o sobrediagnóstico é realmente difícil e poucos estudos seguiram uma boa metodologia. O melhor exemplo é o Malmö Mammographic Screening Trial, cuja taxa de sobrediagnóstico estimada foi de 10\%. Todos os estudos que estimaram de forma correta a taxa de sobrediagnóstico encontraram valores iguais ou inferiores a 10\%. 
TABELA 5. Meta-análises incluídas na revisão.

META-ANÁLISE

Irvin VL, Kaplan RM (2014) $)^{11}$

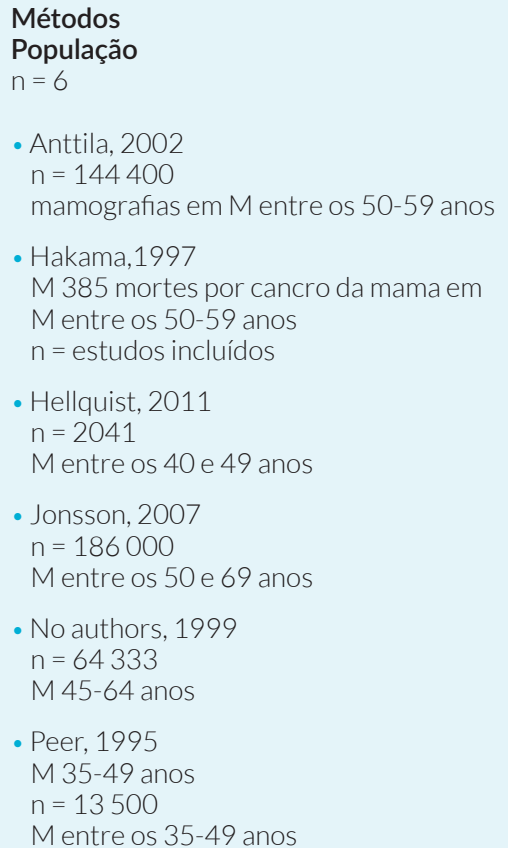

\section{Resultados}

Impacto da mamografia de rastreio na diminuição da mortalidade por cancro da mama contra a não realização da mamografia de rastreio

Anttila (2002): redução de 19\% da mortalidade nas mulheres que realizaram mamografia de rastreio.

Hakama (1997): mostrou que o rastreio do cancro da mama por mamografia previne uma morte por cada 10000 rastreios.

Hellquist (2011): mostrou uma redução da mortalidade estatisticamente significativa no grupo que realizou mamografia de rastreio. Para o grupo com idades compreendidas entre os 40 e os 44 anos, a redução estimada da mortalidade por cancro da mama foi menor do que a do grupo com idades entre 45 e 49 anos.

Jonsson (2007): Este estudo indica uma redução de longo prazo da mortalidade por cancro da mama em 30\%-35\%.

No authors (1999) redução da taxa de mortalidade por cancro da mama no grupo de mulheres rastreadas de $27 \%$, em 16 anos de seguimento.

Peer (1995): não demonstrou uma redução estatisticamente significativa na taxa de mortalidade no grupo de mulheres que realizou mamografia de rastreio.

\section{Sobrediagnóstico e sobretratamento das mulheres submetidas a mamografia de ras- treio}

Nos vários artigos analisados, não foram encontrados dados referentes a este ponto.

\section{Conclusão}

O rastreio com mamografia produz um efeito modesto na mortalidade por cancro da mama, com maior benefício na faixa etária entre os 50 e os 69 anos.

\section{Intervenção}

Mamografia de rastreio de cancro da

mama

Nickson C, Mason KE, English DR, et al (2012) ${ }^{19}$

Meta-análise de $\mathrm{N}=9$ e um estudo caso-controlo baseado no programa de rastreio BreastScreen da Austrália Ocidental

\section{Intervenção}

Mulheres que faleceram por cancro da mama e mulheres vivas sem doença; mamografia de rastreio versus sem mamografia
A participação no programa BreastScreen da Austrália Ocidental (mamografia bienal), na faixa etária de 50 a 69 anos, foi associada a uma mortalidade estimada de $52 \%$ mais baixa por cancro da mama.

A meta-análise foi realizada no sentido de testar a hipótese de que a participação em programas de mamografia de rastreio está associada a uma redução na mortalidade por cancro de mama. A meta-análise dos resultados deste e de outros 9 estudos analisados neste artigo indica que, em média, a participação na mamografia de rastreio foi associada a uma mortalidade $49 \%$ menor por cancro de mama.

\section{NE}

1 
TABELA 6. Ensaio clínico incluído na revisão.

\section{ENSAIO CLÍNICO}

Tabár L, Vitak B, Chen TH, et al (2011)12

Métodos

População

$n=133065$

M 40-74 anos

Seguimento de 29 anos
Resultados

Impacto da mamografia de rastreio na diminuição da mortalidade por cancro da mama contra a não realização da mamografia de rastreio

Houve uma redução significativa na mortalidade por cancro da mama na população rastreada $(R R=0,69$ 95\% IC: 0,56, 0,84; p,0001). Foram salvos 42 anos de vida por 1000 mulheres rastreadas.

O estudo original demonstrou 30\% de redução na mortalidade, valor que se manteve nestes 29 anos de seguimento.

Após 29 anos de seguimento, o número de mulheres necessário rastrear durante 7 anos para prevenir uma morte por cancro da mama foi 414 (segundo dados locais) e 519 (dados de consenso). São necessárias 1334 (dados locais) ou 1677 (dados de consenso) mamografias de rastreio para evitar 1 morte.

Sobrediagnóstico e sobretratamento das mulheres submetidas a mamografia de rastreio

$\mathrm{O}$ artigo não tem dados referentes a este ponto.

( $R R$ - risco relativo; $I C$ - intervalo de confiança; $M$ - mulheres; $n$ - tamanho da amostra)

\section{DISCUSSÃO}

As RS apresentadas classificam-se com um elevado nível de evidência. Em relação ao impacto da mamografia de rastreio na diminuição da mortalidade por cancro da mama, as RS incluídas nesta revisão não são consistentes entre si, sendo que duas delas apontam para o benefício do rastreio ${ }^{13,14}$ e uma outra concluiu que não houve evidência do benefício do rastreio. ${ }^{6}$

Os estudos de coorte retrospetivos ${ }^{15-17}$ incluídos são metodologicamente robustos, tendo um tamanho amostral adequado e um período de seguimento entre os 8,917 e os $17^{15}$ anos. Os seus resultados foram consistentes no que toca à redução da mortalidade por cancro da mama no grupo de mulheres rastreadas, embora Kalager et al ${ }^{17}$ refira que a magnitude deste benefício parece ser modesta.

A redução da mortalidade não pode ser apenas explicada pela realização da mamografia, mas deve ser entendido em contexto multifatorial. Em primeiro lugar os rastreios, ao aumentarem a consciencialização da população para os problemas de saúde, levam potencialmente a um aumento das atitudes preventivas e a medidas de deteção precoce (autoexame, por exemplo). Outro aspeto indissociável à redução das taxas de mortalidade é a melhoria do tratamento, quando este é muito eficaz, independentemente da sintomatologia apresentada, o rastreio deixa de ter um papel importante. ${ }^{18}$

O facto da não inclusão dos estudos históricos na nossa revisão, vem precisamente tentar diluir o efeito das mudanças ao nível das condições sócio/económico/habitacionais verificadas ao longo dos tempos, bem como tentar esbater o impacto da evolução verificada ao nível dos tratamentos médicos na mortalidade por cancro da mama, aspetos que, se não fossem acautelados, poderiam criar um viés em termos dos resultados obtidos.
O estudo de caso-controlo,, classificado com um nível 2 de evidência, teve um período de seguimento de 25 anos e um adequado tamanho amostral ( $n=89$ 835). Este concluiu que a mamografia de rastreio não reduz a mortalidade por cancro da mama. Podemos afirmar que é um estudo robusto pelo significativo período de seguimento, no sentido em que períodos de seguimento muito reduzidos podem originar viés nos resultados obtidos.

A revisão clássica incluída ${ }^{8}$ não descreve que haja benefício da mamografia da redução da mortalidade.

Relativamente ao sobrediagnóstico e sobretratamento das mulheres submetidas a mamografia de rastreio, as RS incluídas nesta revisão foram consistentes a demonstrar uma maior taxa de sobrediagnóstico e sobretratamento. A RS de Nelson et $a^{14}$ acrescenta que existe uma maior probabilidade de falsos positivos nas mulheres mais jovens (40-49 anos). Em contrapartida, a RS de Broeders et $a^{133}$ sugere que quantos mais anos de rastreio maior será o seu benefício na mortalidade por cancro da mama.

A radioterapia está associada a um aumento de mortalidade por doença cardíaca e por cancro de pulmão. $\bigcirc$ estudo de caso-controlo ${ }^{7}$ concluiu que $22 \%$ dos cancros de mama detetados por mamografia são sobrediagnosticados e sobretratados. É de elevada importância valorizar o sobretratamento uma vez que este é prejudicial. Para reforçar esta ideia, Ann Johnson, ${ }^{8}$ na sua revisão clássica, também demonstra que existe um aumento do diagnóstico de carcinomas de mama indolentes, o que tornam o rastreio do cancro da mama menos apelativo.

\section{CONCLUSÃO}

Os estudos analisados nesta revisão baseada na evidência são na sua maioria metodologicamente robustos e de boa qualidade, salientando-se as revisões sistemáticas 
(uma das quais da Cochrane) e a meta-análise. De uma forma geral, os estudos apontam para uma redução da mortalidade por cancro da mama nas mulheres submetidas a mamografia de rastreio com idades compreendidas entre os 50 e os 69 anos de idade. No entanto, não se observa uma diminuição da mortalidade global, aspeto fundamental de um rastreio organizado. Desta forma, em todos os estudos analisados colocam em causa a continuidade dos programas de rastreio organizados de cancro da mama, constituindo esta uma força de recomendação A.

Parece consensual na literatura a evidência de sobrediagnóstico provocado pelos programas de rastreio de cancro da mama cujas implicações não podem ser menosprezadas, nomeadamente o sobretratamento. No entanto, na faixa etária dos 50-69 anos, o benefício da realização deste rastreio, objetivável pela redução da mortalidade, parece ser superior aos seus eventuais riscos. ${ }^{11}$

Tem-se constatado, em alguns países da união europeia, recomendações acerca da abolição ou redução do rastreio do cancro da mama organizado, com base em estudos de elevada qualidade metodológica realizados no próprio país. ${ }^{6}$ Para extrapolar à nossa população, seriam necessários estudos nacionais com elevada robustez.

CONFLITOS DE INTERESSE: Os autores declaram não ter qualquer conflito de interesse na realização do presente trabalho.

FONTES DE FINANCIAMENTO: Não houve qualquer fonte de financiamento na realização do presente trabalho.

CONFLICTS OF INTEREST: The authors declare that they have no conflicts of interest.

FINANCIAL SUPPORT: This work has not received any contribution, grant or scholarship.

\section{REFERÊNCIAS}

1. Jørgensen KJ, Keen JD, Gøtzsche PC. Is mammographic screening justifiable considering its substantial overdiagnosis rate and minor effect on mortality? Radiology. 2011;260:621-7. doi:10.1148/radiol.11110210.

2. Utzon-Frank N, Vejborg I, von Euler-Chelpin M, Lynge E. Balancing sensitivity and specificity: Sixteen year's of experience from the mammography screening programme in Copenhagen, Denmark. Cancer Epidemiol. 2011;35:393-8. doi:10.1016/j.canep.2010.12.001.

3. Biller-andorno N, Jüni P. Abolishing mammography screening programs? a view from the Swiss Medical Board. 2014:474-5.

4. Review C, Communication S. Quantifying the Benefits and Harms of Screening Mammography. JAMA Intern Med. 2014;174:448-54. doi:10.1001/jamainternmed.2013.13635.

5. Ebell M, Siwek J, Weiss B. Strength of Recommendation Taxonomy (SORT) Fam Med. 2004;36:141-3.
6. Gotzsche PC, Jørgensen K. Screening for breast cancer with mammography. Cochrane Database Syst Rev. 2013:CD001877. doi:10.1002/14651858.CD001877.pub5. www.cochranelibrary.com.

7. Miller AB, Wall C, Baines CJ, Sun P, To T, Narod SA. Twenty five year follow-up for breast cancer incidence and mortality of the Canadian National Breast Screening Study: randomised screening trial.BMJ. 2014;366:1-10. doi:10.1136/bmj.g366.

8. Johnson A. "Overdiagnosis " and mortality in breast cancer screening. J R Soc Med. 2012;105:317-9. doi:10.1258/ jrsm.2012.120003.

9. Mayor S. Mammography screening has little or no effect on breast cancer deaths, Swedish data indicate. BMJ. 2012;345:e4847-e4847. doi:10.1136/bmj.e4847.

10. Kopans DB, Smith R, Duffy SW. Mammographic screening and "overdiagnosis". Radiology. 2011;260:616-20. doi:10.1148/ radiol.11110716.

11. Irvin VL, Kaplan RM. Screening mammography \& breast cancer mortality: meta-analysis of quasi-experimental studies. PLoS One. 2014;9(:e98105. doi:10.1371/journal.pone.0098105.

12. Tabar L, Vitak B, Chen T, Yen AM, Cohen A, Tot T, et al. Swedish two-county trial: impact of mammographic screening on breast cancer mortality during 3 decades - with comments. Radiology. 2011;260:658-63. doi:10.1148/radiol.11110469.

13. Broeders M, Moss S, Nystrom L, Njor S, Jonsson H, Paap E, et al. The impact of mammographic screening on breast cancer mortality in Europe: a review of observational studies. J Med Screen. 2012;19 Suppl 1:14-25.

14. Nelson H, Tyne K, Naik A, Bougatsos C, Nygren P, Humphrey L. Screening for breast cancer: systematic evidence review update for the U . S. Preventive Services Task Force. Rockville: Agency for Healthcare Research and Quality; 2010.

15. Kaplan HG, Malmgren JA, Atwood MK, Calip GS. Effect of treatment and mammography detection on breast cancer survival over time: 1990-2007. Cancer. 2015;121:2553-2561. doi:10.1002/cncr.29371.

16. Hofvind S, Ursin G, Tretli S, Sebuødegård S, Møller B. Breast cancer mortality in participants of the Norwegian Breast Cancer Screening Program. Cancer. 2013;119:3106-112. doi:10.1002/cncr.28174.

17. Mette Kalager, Zelen M, Langmark F, Adami H-O. Effect of screening mammography on breast-cancer mortality in Norway. New Engl J Med. 2010:1991-2002. doi:10.1056/NEJMoa1414264.

18. Burke JP, Barry M, Kell MR. More on screening mammography. N Engl J Med. 2011;364:281-6. doi:10.1056/NEJMc1011881.

19. Nickson C, Mason KE, English DR, Kavanagh AM. Mammographic screening and breast cancer mortality: a case - control study and meta-analysis. Cancer Epidemiol Biomarkers Prev. 2012;21:1479-88. doi:10.1158/1055-9965.EPI-12-0468. 